PROCEEDINGS OF THE

AMERICAN MATHEMATICAL SOCIETY

Volume 138, Number 9, September 2010, Pages 3335-3343

S 0002-9939(10)10415-8

Article electronically published on April 29, 2010

\title{
MAX-MIN CHARACTERIZATION OF THE MOUNTAIN PASS ENERGY LEVEL FOR A CLASS OF VARIATIONAL PROBLEMS
}

\author{
JACOPO BELLAZZINI AND NICOLA VISCIGLIA \\ (Communicated by Matthew J. Gursky)
}

\begin{abstract}
We prove the existence of a critical point at the mountain pass energy level for a general class of variational problems. We also provide a Max-Min characterization of the mountain pass energy level. Finally, we present some concrete applications.
\end{abstract}

In the literature the existence of solutions for variational PDEs is often reduced to the existence of critical points of functionals $F$ having the following structure:

$$
F(u)=T(u)-U(u),
$$

where $u$ belong to a Banach space $X$ and $T, U$ satisfy suitable conditions.

A classical strategy to find critical points for the functional $F$ is to look at the constrained minimization problem

$$
\min _{U(u)=1} T(u)
$$

and eventually to remove the Lagrange multiplier that appears by using suitable invariances of the functional.

On the other hand, when the functional $F$ has a mountain pass geometry it is customary to look at critical points of the unconstrained functional $F(u)$ on the whole space $X$. More specifically it is well known that a candidate critical value of $F$ is given by the mountain pass energy level:

$$
c:=\inf _{\eta \in \Gamma} \sup _{t \in[0,1]} F(\eta(t)),
$$

where

$$
\Gamma:=\{\eta \in C([0,1] ; X \mid \eta(0)=0, F(\eta(1))<0\} .
$$

A natural question is to ask whether or not the two forementioned approaches provide the same solution. In both approaches described above the main difficulty is related to the lack of compactness respectively for the minimizing sequences and for the Palais Smale sequences.

Received by the editors June 24, 2009 and, in revised form, September 4, 2009 and December 16, 2009.

2000 Mathematics Subject Classification. Primary 58E05; Secondary 35J20, 35J60.

Key words and phrases. Critical point theory, mountain pass energy level, nonlinear elliptic equations.

(C)2010 American Mathematical Society Reverts to public domain 28 years from publication 3335 
The aim of this paper is twofold: we prove the existence of a critical point at the mountain pass energy level by looking at a suitable family of constrained minimization problems, and we also give a Max-Min characterization of the mountain pass value $c$ in terms of the energy of the corresponding constrained minimizers. More precisely we relate the existence of a regular path of minimizers for

$$
\min _{U(u)=\lambda} T(u), \lambda \in \mathbb{R}^{+}
$$

with the existence of a critical point for $F(u)$ having the mountain pass energy level.

Let us fix some notation. We shall denote by $X^{\prime}$ the topological dual of the Banach space $\mathrm{X}$ and by $(., .)_{X^{\prime}, X}$ the usual duality between $X$ and $X^{\prime}$. For every $\lambda \in \mathbb{R}$ we introduce the sets

$$
\mathcal{U}_{\lambda}:=\{u \in X \mid U(u)=\lambda\}
$$

and

$$
i_{\lambda}:=\inf _{u \in \mathcal{U}_{\lambda}} T(u)
$$

Finally we introduce

$$
\mathcal{M}_{\lambda}:=\left\{u \in \mathcal{U}_{\lambda} \mid T(u)=i_{\lambda}\right\}
$$

(notice that it could even be the empty set).

Theorem 0.1. Let $X$ be a Banach space and $F=T-U$ with:

$$
\begin{gathered}
T, U \in \mathcal{C}(X, \mathbb{R}), T(u) \geq 0, T(0)=U(0)=0 ; \\
\lim _{\lambda \rightarrow 0^{+}} i_{\lambda}=0 ; \\
\lambda^{*}>0, \text { where } \lambda^{*}:=\inf \left\{\lambda \in(0, \infty) \text { such that } i_{\lambda}-\lambda \leq 0\right\} ; \\
\lambda^{* *}>0, \text { where } \lambda^{* *}:=\inf \left\{\lambda \in(0, \infty) \text { such that } i_{\lambda}-\lambda<0\right\} ; \\
\mathcal{M}_{\lambda} \neq \emptyset \forall \lambda \in \mathbb{R}^{+} ; \\
\text {there exists a continuous map } \gamma:[0, \infty) \rightarrow X \\
\text { such that } \gamma(\lambda) \in \mathcal{M}_{\lambda} .
\end{gathered}
$$

Then $c>0$ and

$$
\max _{\lambda \in\left(0, \lambda^{* *}\right)}\left(\min _{u \in \mathcal{U}_{\lambda}} F(u)\right)=\max _{\lambda \in\left(0, \lambda^{* *}\right)} F(\gamma(\lambda))=c,
$$

where $c$ is defined in (0.1). Assume moreover that $F \in \mathcal{C}^{1}(X, \mathbb{R})$. Then $c$ is a critical value for $F$, and in particular the set

$$
\mathcal{K}=\{\gamma(\lambda) \in X \mid F(\gamma(\lambda))=c\}
$$

contains at least a critical point of the functional $F$.

Remark 0.1. The hypothesis (0.8) concerns a continuous selection of minima with respect to the parameter $\lambda$. Typically this is the hardest condition to be concretely checked. However if suitable invariances of the variational problem are available, then this property is satisfied (see Corollaries 0.1 and 0.2 ).

Remark 0.2. Notice that by definition we have the following identity:

$$
F(\gamma(\lambda))=i_{\lambda}-\lambda \forall \lambda>0 .
$$


Hence the set $\mathcal{K}$ given in $(0.10)$ can be defined in an equivalent way as follows:

$$
\left\{\gamma(\lambda) \in X \mid i_{\lambda}-\lambda=c, \lambda>0\right\} \text {. }
$$

Next we give two concrete applications of our general result, based on the following invariances:

$$
\begin{gathered}
\text { rescaling: } u \rightarrow u\left(\frac{x}{\beta}\right), \\
\text { contraction/ expansion: } u \rightarrow \beta u(x) .
\end{gathered}
$$

The model equation for rescaling is given by

$$
-\Delta_{p} u-\frac{\mu}{|x|^{p}}|u|^{p-2} u=g(u) \text { on } \mathbb{R}^{n}, n \geq 3,
$$

which is the Euler-Lagrange equation corresponding to the following functional:

$$
W^{1, p}\left(\mathbb{R}^{n}\right) \ni u \rightarrow F(u):=\frac{1}{p} \int\left(|\nabla u|^{p}-\frac{\mu}{|x|^{p}}|u|^{p}\right) d x-\int G(u) d x .
$$

Next we fix the following specific framework:

$$
\begin{gathered}
X:=W^{1, p}\left(\mathbb{R}^{n}\right), \\
T(u):=\frac{1}{p} \int\left(|\nabla u|^{p}-\frac{\mu}{|x|^{p}}|u|^{p}\right) d x \text { with } 0 \leq \mu<\left(\frac{n-p}{p}\right)^{p}, \\
U(u):=\int G(u) d x .
\end{gathered}
$$

Following the same arguments as in [1] for $p=2$ and $[3$ for $p \neq 2$, it is easy to deduce that (0.7) (i.e. the existence of a minimizer for $T(u)$ constrained on $U(u)=\lambda$ ) holds in the specific context given in (0.14) provided that $1<p<N$ and the nonlinearity $g$ fulfills:

$$
\begin{gathered}
g(s) \in \mathcal{C}(\mathbb{R}, \mathbb{R}) \text { is continuous and odd; } \\
-\infty<\liminf _{s \rightarrow 0} \frac{g(s)}{s} \leq \limsup _{s \rightarrow 0} \frac{g(s)}{s}<0 ; \\
\lim _{s \rightarrow \infty} \frac{g(s)}{s^{p^{*}-1}}=0, \text { where } p^{*}=\frac{n p}{n-p} ; \\
\text { there exists } \xi_{0}>0 \text { s.t. } G\left(\xi_{0}\right)>0,
\end{gathered}
$$

obtained by scaling the minimizers described above to coincide with the mountain pass energy level. Let us emphasize that $F, c, \mathcal{M}_{1}$ and $i_{1}$ are defined as in Theorem 0.1 in the concrete situation given by (0.14).

Corollary 0.1. Assume $1<p<n$, G satisfies (0.15)-(0.16)-(0.17)-(0.18), $v \in \mathcal{M}_{1}$ and

$$
\bar{\lambda}=\left(i_{1}\right)^{\frac{n}{p}}\left(\frac{n-p}{p}\right)^{\frac{n}{p}} .
$$

Then $u=v\left(\frac{x}{\bar{\lambda}^{\frac{1}{n}}}\right)$ is a solution of (0.13) whose energy level is given by the mountain pass energy level $c$. Moreover the path $\gamma:(0, \infty) \rightarrow X$ given by

$$
\lambda \rightarrow v\left(\frac{x}{\lambda^{\frac{1}{n}}}\right)
$$

satisfies $\max _{\lambda \in(0, \infty)} F(\gamma(\lambda))=c$ and $v\left(\frac{x}{\bar{\lambda}^{\frac{1}{n}}}\right)$ is the least energy solution among all the solutions $u$ of (0.13) belonging to the set $\left\{u \mid \int G(u) d x>0\right\}$. 
Remark 0.3. Let us recall that in the specific case $\mu=0$ the Pohozaev identity guarantees that any solution of (0.13) belongs to the set $\left\{u \mid \int G(u) d x>0\right\}$. Therefore in the case $\mu=0$ we recover the results contained in [5] and [3] concerning the identification between the mountain pass energy level with the least energy level.

Our second application concerns the contraction-expansion scale invariance. We consider the equation

$$
\left\{\begin{array}{l}
-\Delta_{p} u-\mu|u|^{p-2} u=u|u|^{p^{*}-2} u \text { in } \Omega \subset \mathbb{R}^{n} \\
u=0 \text { on } \partial \Omega
\end{array}\right.
$$

with

$$
\begin{gathered}
p^{*}=\frac{n p}{n-p}, \Omega \text { is open and bounded, } 1<p^{2}<n, \\
0<\mu<\mu_{p},
\end{gathered}
$$

where

$$
\mu_{p}=\inf _{u \neq 0} \frac{\int|\nabla u|^{p} d x}{\int|u|^{p} d x} .
$$

This equation is the Euler-Lagrange equation corresponding to the following functional:

$$
W_{0}^{1, p}(\Omega) \ni u \rightarrow F(u):=\frac{1}{p} \int\left(|\nabla u|^{p}-\mu|u|^{p}\right) d x-\frac{1}{p^{*}} \int u^{p^{*}} d x .
$$

Next we fix the following specific framework:

$$
\begin{gathered}
X:=W_{0}^{1, p}(\Omega), \\
T(u):=\frac{1}{p}\left(\int|\nabla u|^{p}-\mu|u|^{p} d x\right), \\
U(u):=\frac{1}{p^{*}} \int|u|^{p^{*}} d x .
\end{gathered}
$$

The validity of hypothesis (0.7) in this specific framework has been checked in 2] for $p=2$ and in 4 for $p \neq 2$. In the next corollary, $F, c, \mathcal{M}_{1}$ and $i_{1}$ are defined as in Theorem 0.1 in the concrete situation given by (0.21).

Corollary 0.2. Let (0.20) hold, $v \in \mathcal{M}_{1}$ and $\bar{\lambda}=\left(\frac{i_{1} p}{p^{*}}\right)^{\frac{p^{*}}{p^{*}-p}}$. Then $u=\bar{\lambda}^{\frac{p}{p^{*}}} v$ is a solution of (0.19) whose energy level is given by the mountain pass energy level. Moreover the path $\gamma:(0, \infty) \rightarrow X$ given by

$$
\lambda \rightarrow \lambda^{\frac{p}{p^{*}}} v
$$

satisfies $\max _{\lambda \in(0, \infty)} F(\gamma(\lambda))=c$, and $u=\bar{\lambda}^{\frac{p}{p^{*}}} v$ is the least energy solution among all the solutions of (0.19).

\section{Proof of Theorem 0.1}

Proof of (0.9). Notice that $(0, \infty) \ni \lambda \rightarrow I_{\lambda}:=i_{\lambda}-\lambda$ is a continuous function due to (0.3) and (0.8). Let

$$
I:=\max _{\lambda \in\left(0, \lambda^{* *}\right)} I_{\lambda} \text { and } \mathcal{A}=\left\{\lambda \in\left(0, \lambda^{* *}\right) \text { such that } I_{\lambda}=I\right\} .
$$

We claim the following fact:

Given $\eta \in \Gamma$ there is $\bar{t}$ such that $\eta(\bar{t}) \in \mathcal{U}_{\bar{\lambda}}$, where $\bar{\lambda} \in \mathcal{A}$ (recall that $\Gamma$ is defined in $(\underline{0.2)})$. 
We show how the claim implies (0.9). We have

$$
\max _{t \in[0,1]} F(\eta(t)) \geq F(\eta(\bar{t}))=T(\eta(\bar{t}))-U(\eta(\bar{t})) \geq i_{\bar{\lambda}}-\bar{\lambda} ;
$$

hence

$$
c \geq i_{\bar{\lambda}}-\bar{\lambda}=I=\max _{\lambda \in\left(0, \lambda^{* *}\right)} F(\gamma(\lambda)),
$$

where $\gamma \in \Gamma$ is defined by (0.8). By definition of $\lambda^{* *}$ there is a sequence $\lambda_{n}>\lambda^{* *}$ such that $\lambda_{n} \rightarrow \lambda^{* *}, F\left(\gamma\left(\lambda_{n}\right)\right)<0$ and moreover $\lim _{n \rightarrow \infty} F\left(\gamma\left(\lambda_{n}\right)\right)=0$. As a consequence there is $\bar{n} \in \mathbb{N}$ such that

$$
\sup _{\lambda \in\left(0, \lambda_{\bar{n}}\right)} F(\gamma(\lambda))=\sup _{\lambda \in\left(0, \lambda^{* *}\right)} F(\gamma(\lambda)) .
$$

In particular, after a suitable parametrization, $\gamma:\left[0, \lambda_{\bar{n}}\right] \rightarrow X$ belongs to $\Gamma$ and hence

$$
c \leq \max _{\lambda \in\left(0, \lambda^{* *}\right)} F(\gamma(\lambda)) .
$$

By combining this fact with (1.1) we get $c=\max _{\lambda \in(0, \lambda * *)} F(\gamma(\lambda))$.

In order to prove the claim stated above we notice that (0.5) implies that

$$
F(u) \geq 0 \forall u \in \bigcup_{\lambda \in\left(-\infty, \lambda^{* *}\right]} \mathcal{U}_{\lambda}
$$

and since $\eta \in \Gamma$, necessarily

$$
\eta(1) \in \mathcal{U}_{\lambda} \text { with } \lambda>\lambda^{* *} .
$$

Next consider the continuous function

$$
(0,1) \ni t \rightarrow U(\eta(t)) .
$$

By combining the definition of $\Gamma$ with (1.2) we get $U(\eta(0))=0$ and $U(\eta(1))>\lambda^{* *}$; hence by a continuity argument we have the claim since $0 \leq \bar{\lambda} \leq \lambda^{* *}$.

Proof of the existence of a critical point in $\mathcal{K}$. Let us fix $\bar{\lambda}>\lambda^{* *}$ such that $F(\gamma(\bar{\lambda}))$ $<0$ and

$$
c=\sup _{\left[0, \lambda^{* *}\right]} F(\gamma(\lambda))=\sup _{[0, \bar{\lambda}]} F(\gamma(\lambda)) .
$$

Next we shall prove that the set

$$
\mathcal{K}^{\prime}=\{\gamma(\lambda) \in X \mid F(\gamma(\lambda))=c \text { and } \lambda \in[0, \bar{\lambda}]\}
$$

contains a critical point. Assuming by the absurd that it is not true, then (since $\mathcal{K}^{\prime}$ is compact)

$$
\|d F(k)\|_{X^{\prime}} \geq \eta>0 \forall k \in \mathcal{K}^{\prime} .
$$

Moreover there exist two open sets $\mathcal{U}, \mathcal{V}$ and a Lipshitz function $\chi$ valued in $[0,1]$ such that

$$
\begin{gathered}
\mathcal{K}^{\prime} \subset \mathcal{U} \subset \overline{\mathcal{U}} \subset \mathcal{V} \subset X, \\
\|d F(v)\|_{X^{\prime}} \geq \frac{\eta}{2}>0 \forall v \in \mathcal{V}, \\
\mathcal{V} \cap\{\gamma(0), \gamma(\bar{\lambda})\}=\emptyset, \\
\chi \equiv 1 \text { on } \mathcal{U} \text { and }\{x \in X \mid \chi(x)=0\}=X \backslash \mathcal{V} .
\end{gathered}
$$


Next we introduce a locally Lipschitz pseudo-gradient vector field $Y \in \mathcal{C}^{1}(X, X)$ such that

$$
\|Y(x)\|_{X} \leq 2\|d F(x)\|_{X^{\prime}} \forall x \in X
$$

and

$$
(d F(x), Y(x))_{X^{\prime}, X} \geq\|d F(x)\|_{X^{\prime}}^{2}, \forall x \in X
$$

(see e.g. 6] for a proof of the existence of $Y$ ).

Notice that by combining (1.5) with (1.8) we get

$$
\|Y(v)\|_{X} \geq \frac{\eta}{2}>0 \forall v \in \mathcal{V} .
$$

By combining (1.9) with (1.7) it is easy to deduce that the solutions to the following ODE are globally defined for every $t \in(-\infty, \infty)$ :

$$
\left\{\begin{array}{l}
\frac{d}{d t} \eta(t, x)=-\chi(\eta(t, x)) \frac{Y(\eta(t, x))}{\|Y(\eta(t, x))\|_{X}^{2}}, \\
\eta(0, x)=x
\end{array}\right.
$$

and moreover

$$
\begin{aligned}
& F(\eta(t, x))=F(x) \forall x \in X \backslash \mathcal{V}, t \in \mathbb{R}, \\
& F(\eta(t, x))>F(\eta(s, x)) \forall x \in \mathcal{V}, t<s .
\end{aligned}
$$

Indeed (1.12) follows from the following computation:

$$
\frac{d}{d t} F(\eta(t, x))=\left(d F(\eta(t, x)),-\chi(\eta(t, x)) \frac{Y(\eta(t, x))}{\|Y(\eta(t, x))\|_{X}^{2}}\right)_{X^{\prime}, X}<0 \forall x \in \mathcal{V}, t \in \mathbb{R},
$$

where we have used (1.8) and (1.7). As a consequence of (1.6) and (1.7) the path

$$
\tilde{\gamma}:[0, \bar{\lambda}] \ni \lambda \rightarrow \eta(1, \gamma(\lambda)) \in X
$$

satisfies the following properties:

$$
\tilde{\gamma}(0)=\gamma(0)=0, F(\tilde{\gamma}(\bar{\lambda}))=F(\gamma(\bar{\lambda}))
$$

and in particular (up to a reparametrization) $\tilde{\gamma} \in \Gamma$. Next we prove that

$$
\sup _{[0, \bar{\lambda}]} F(\tilde{\gamma}(\lambda))<c,
$$

and in this way we conclude the proof since this is in contradiction with the definition of the mountain pass energy level $c$. In order to prove (1.13) we fix $\lambda_{0} \in[0, \bar{\lambda}]$ such that

$$
F\left(\tilde{\gamma}\left(\lambda_{0}\right)\right)=\max _{[0, \bar{\lambda}]} F(\tilde{\gamma}(\lambda)) .
$$

There are two possibilities: either $\gamma\left(\lambda_{0}\right) \in X \backslash \mathcal{V}$ or $\gamma\left(\lambda_{0}\right) \in \mathcal{V}$.

In the first case we can deduce by (1.11), (1.4) and by the definition of $\mathcal{K}^{\prime}$ that $F\left(\tilde{\gamma}\left(\lambda_{0}\right)\right)=F\left(\eta\left(1, \gamma\left(\lambda_{0}\right)\right)\right)=F\left(\gamma\left(\lambda_{0}\right)\right)<c$.

In the second case we can deduce by (1.12) that $F\left(\tilde{\gamma}\left(\lambda_{0}\right)\right)=F\left(\eta\left(1, \gamma\left(\lambda_{0}\right)\right)\right)<$ $\left.F\left(\gamma\left(\lambda_{0}\right)\right)\right) \leq c$, where in the last step we have used $\sup _{[0, \bar{\lambda}]} F(\gamma(\lambda))=c$.

Hence (1.13) is proved. 


\section{Applications}

Proof of Corollary 0.1, By the standard Hardy inequality we have that

$$
\left(\frac{n-p}{p}\right)^{p} \int \frac{|u|^{p}}{|x|^{p}} d x \leq \int|\nabla u|^{p} d x
$$

and hence

$$
\int\left(|\nabla u|^{p}-\frac{\mu}{|x|^{p}}|u|^{p}\right) d x
$$

is equivalent to the standard seminorm $\int|\nabla u|^{p} d x$ provided that $\mu$ is as in the assumptions. Moreover, due to the positivity of $\mu$ we can deduce via a rearrangement argument that the minimizing sequences for $i_{1}$ can be chosen to be radially symmetric. Following the same argument as in [1] for $p=2$ and more generally for $p \neq 2$ and [3], we have that $\mathcal{M}_{1} \neq \emptyset$. In order to prove Corollary 0.1 we check first that the general hypotheses of Theorem 0.1 are fulfilled in the specific framework defined in (0.14). Notice that if $u \in \mathcal{U}_{1}$, then $u\left(\frac{x}{\lambda^{\frac{1}{n}}}\right) \in \mathcal{U}_{\lambda}$ and that

$$
T\left(u\left(\frac{x}{\lambda^{\frac{1}{n}}}\right)\right)=\lambda^{1-\frac{p}{n}} T(u) .
$$

Hence we have

$$
i_{\lambda}=\lambda^{1-\frac{p}{n}} i_{1}
$$

and a family of minimizers for $i_{\lambda}$ is given by $v\left(\frac{x}{\lambda^{\frac{1}{n}}}\right)$, where $v \in \mathcal{M}_{1}$. We have

$$
i_{\lambda}-\lambda=\lambda^{1-\frac{p}{n}} i_{1}-\lambda
$$

and thus hypotheses (0.4)-(0.5)-(0.6)-(0.8) are fulfilled. We have

$$
\lambda^{*}=\lambda^{* *}=i_{1}^{\frac{n}{p}} .
$$

An easy computation shows that $\frac{d}{d \lambda}\left(i_{\lambda}-\lambda\right)=0$ for $\bar{\lambda}=\left(i_{1}\right)^{\frac{n}{p}}\left(\frac{n-p}{p}\right)^{\frac{n}{p}}$ and that $\bar{\lambda}$ fulfills

$$
\bar{\lambda}^{1-\frac{p}{n}} i_{1}-\bar{\lambda}=\max _{\lambda \in(0, \infty)} \lambda^{1-\frac{p}{n}} i_{1}-\lambda=\left(i_{1}\right)^{\frac{n}{p}}\left(\left(\frac{n-p}{p}\right)^{\frac{n}{p}-1}-\left(\frac{n-p}{p}\right)^{\frac{n}{p}}\right) .
$$

In particular by Remark (0.2) the set $\mathcal{K}$ (see (0.10) in Theorem 0.1 ) reduces in this specific context to the point $v\left(\frac{x}{\bar{\lambda}^{\frac{1}{n}}}\right)$, and hence it is a solution of (0.13) such that

$$
F\left(v\left(\frac{x}{\bar{\lambda}^{\frac{1}{n}}}\right)\right)=\left(i_{1}\right)^{\frac{n}{p}}\left(\left(\frac{n-p}{p}\right)^{\frac{n}{p}-1}-\left(\frac{n-p}{p}\right)^{\frac{n}{p}}\right)=c .
$$

Now we prove that $v\left(\frac{x}{\bar{\lambda}^{\frac{1}{n}}}\right)$ is the least energy solution among all solutions of (0.13) belonging to the set $\left\{u \mid \int G(u) d x>0\right\}$. Letting $\psi$ be another solution of (0.13), we show that $F(\psi) \geq F\left(v\left(\frac{x}{\bar{\lambda}^{\frac{1}{n}}}\right)\right)$. Notice that if $\psi$ is a solution of (0.13) with $\psi \in \mathcal{U}_{\lambda_{1}}$ with $\lambda_{1}>0$, we have $\phi\left(\frac{x}{\lambda_{1}^{\frac{1}{N}}}\right)=\psi(x)$ with $\phi \in \mathcal{U}_{1}$. Moreover

$$
\begin{aligned}
& F\left(\phi\left(\frac{x}{\lambda^{\frac{1}{N}}}\right)\right)=\lambda^{1-\frac{p}{n}} T(\phi)-\lambda, \\
& \left.\frac{d}{d \lambda}\left(F\left(\phi\left(\frac{x}{\lambda^{\frac{1}{n}}}\right)\right)\right)\right|_{\lambda=\lambda_{1}}=0 .
\end{aligned}
$$


Hence $\psi$ is critical point iff $\lambda_{1}$ fulfills

$$
\lambda_{1}=(T(\phi))^{\frac{n}{p}}\left(\frac{n-p}{p}\right)^{\frac{n}{p}} .
$$

We obtain $F(\psi)=T(\phi)^{\frac{n}{p}}\left(\left(\frac{n-p}{p}\right)^{\frac{n}{p}-1}-\left(\frac{n-p}{p}\right)^{\frac{n}{p}}\right) \geq F\left(v\left(\frac{x}{\bar{\lambda}^{\frac{1}{n}}}\right)\right)=c$.

Proof of Corollary 0.2 . The fact that $\mathcal{M}_{1} \neq \emptyset$ is proved in [2] and [4] in the case $p=$ 2 and $p \neq 2$ respectively. The proof follows exactly as in the proof of Corollary 0.1 once we notice that if $u \in \mathcal{U}_{1}$, then $\lambda^{\frac{1}{p^{*}}} u(x) \in \mathcal{U}_{\lambda}$ and that

$$
T\left(\lambda^{\frac{1}{p^{*}}} u\right)=\lambda^{\frac{p}{p^{*}}} T(u) .
$$

Hence we have

$$
i_{\lambda}=\lambda^{\frac{p}{p^{*}}} i_{1}
$$

and a path of minimizer for $i_{\lambda}$ is given by $(0, \infty) \ni \lambda \rightarrow \lambda^{\frac{1}{p^{*}}} v$, where $v \in \mathcal{M}_{1}$. We have

$$
i_{\lambda}-\lambda=\lambda^{\frac{p}{p^{*}}} i_{1}-\lambda .
$$

Notice finally that $\frac{d}{d \lambda}\left(i_{\lambda}-\lambda\right)=0$ for $\bar{\lambda}=\left(\frac{i_{1} p}{p^{*}}\right)^{\frac{p^{*}}{p^{*}-p}}$ and that $\bar{\lambda}$ fulfills $\bar{\lambda}^{\frac{p}{p^{*}}} i_{1}-\bar{\lambda}=$ $\max _{\lambda \in(0, \infty)} \lambda^{\frac{p}{p^{*}}} i_{1}-\lambda$. Then as a combination of Remark 0.2 with Theorem 0.1 we can conclude that $\bar{\lambda}^{\frac{p}{p^{*}}} v$ is a solution of (0.19) with energy given by

$$
F\left(\bar{\lambda}^{\frac{p}{p^{*}}} v\right)=\left(i_{1} \frac{p}{p^{*}}\right)^{\frac{p^{*}}{p^{*}-p}}\left(\frac{p^{*}-p}{p}\right)=c .
$$

Notice that if $\psi$ is a solution of (0.19) with $\psi \in \mathcal{U}_{\lambda_{2}}$, we have $\lambda_{2}>0$. Indeed we have

$$
\int|\nabla \psi|^{p}-\mu|\psi|^{p} d x=\int|\psi|^{p^{*}} d x=p^{*} \lambda_{2} .
$$

We have $\lambda_{2}^{\frac{1}{p^{*}}} \phi=\psi(x)$ with $\phi \in \mathcal{U}_{1}$. Moreover

$$
\begin{aligned}
& F\left(\lambda^{\frac{1}{p^{*}}} \phi\right)=\lambda^{\frac{p}{p^{*}}} T(\phi)-\lambda, \\
& \left.\frac{d}{d \lambda}\left(F\left(\lambda^{\frac{1}{p^{*}}} \phi\right)\right)\right|_{\lambda=\lambda_{2}}=0 .
\end{aligned}
$$

Hence $\psi$ is a critical point iff $\lambda_{2}$ fulfills

$$
\lambda_{2}=\left(\frac{T(\phi) p}{p^{*}}\right)^{\frac{p^{*}}{p^{*}-p}} .
$$

We obtain $F(\psi)=\left(T(\phi) \frac{p}{p^{*}}\right)^{\frac{p^{*}}{p^{*}-p}}\left(\frac{p^{*}-p}{p}\right) \geq\left(i_{1} \frac{p}{p^{*}}\right)^{\frac{p^{*}}{p^{*}-p}}\left(\frac{p^{*}-p}{p}\right)=c$.

\section{REFERENCES}

[1] H. Berestycki, P.L. Lions, Nonlinear scalar field equations. I, Arch. Rat. Mech. Anal. 82, 313346 (1983). MR0695535(84h:35054a)

[2] H. Brezis, L. Nirenberg, Positive solutions of nonlinear elliptic equations involving critical Sobolev exponents, Comm. Pure Appl. Math. 36, no. 4, 437-477 (1983). MR709644 (84h:35059)

[3] J.M. do Ó, E. Medeiros, Remarks on least energy solutions for quasilinear elliptic problems in $\mathbb{R}^{N}$, Electron. J. Differential Equations, No. 83 (2003). MR1995609 (2004i:35090)

[4] M. Guedda, L. Veron, Quasilinear elliptic equations involving critical Sobolev exponents, Nonlinear Anal. 13, no. 8, 879-902 (1989). MR1009077 (90h:35100) 
[5] L. Jeanjean, K. Tanaka, A remark on least energy solutions on $\mathbb{R}^{n}$, Proc. Amer. Math. Soc. 138, 2399-2408 (2003). MR 1974637 (2004c:35127)

[6] M. Willem, Minimax theorems, Progress in Nonlinear Differential Equations and Their Applications, 24. Birkhäuser Boston, Inc., Boston, MA, 1996. MR.1400007 (97h:58037)

Dipartimento di Matematica Applicata, Università di Pisa, Via Buonarroti 1/C, 56127 Pisa, ITALY

E-mail address: j.bellazzini@ing.unipi.it

Dipartimento di Matematica, Università di Pisa, Largo B. Pontecorvo 5, 56100 Pisa, ITALY

E-mail address: viscigli@dm.unipi.it 